\title{
Toxicological Indices of Wistar Rats Fed Formulated Chaw of Telfairia occidentalis Planted on Crude Oil Contaminated and Remediated Soil
}

\author{
Reginald C. Ohiri ${ }^{\circledR}$ \\ Eugene N. Onyeike ${ }^{2}$ \\ Augustine A. Uwakwe ${ }^{3}$
}

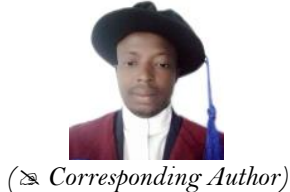

${ }_{0.2,3}$ Department of Biochemistry, Faculty of Science, University of Port Harcourt, Nigeria.

Email: raycohiri@yahoo.com

¿Email: eugene.onyeike@uniport.edu.ng

${ }^{s}$ Email:augustine.uwakwe@uniport.edu.ng

\section{Abstract}

Toxicological indices of wistar rats fed formulated chaw of Telfairia occidentalis planted on crude oil contaminated and bioremediated soil was studied. Farmland, measuring $18 \mathrm{~m}^{2}$ was divided into three lots of $4 \mathrm{~m}^{2}$ and $2 \mathrm{~m}$ space was allowed between each lot (A, B and $\mathrm{C}$ ). Two lots were polluted, subjected to 16 weeks of bioremediation and viable seeds of T. occidentalis were planted and grown for 28 days. The harvested vegetable leaves were analyzed. Leaves from natural attenuated soil showed high concentration of both aliphatic and polycyclic aromatic hydrocarbons with values of $9.34 \pm 0.03 \mathrm{mg} / \mathrm{kg}$ and $9.18 \pm 0.05 \mathrm{mg} / \mathrm{kg}$ for $\mathrm{C}_{36}$ and Fluoranthene respectively, while the bioaugmented soil had Lead as the highest heavy metal with a value of $0.10 \pm 0.02$ $\mathrm{g} / 100 \mathrm{~g}$. Four groups, of 9 rats each (totalling 36 rats) of adult males and females, wealing males and females were subsequently sub-grouped into 3 and allowed to acclimatize for a period of 1 week. These animals were fed formulated rat chaw of $50 \%$ dry weight of $T$. occidentalis, from the three lots (control, bioaugmented and natural attenuated soil) for a period of 28 days. Assay of animals' toxicological index showed elevated concentrations of alkaline phosphatase, alanine and aspartate aminotransferases, urea and creatinine in their sera. A slight increase in serum amylase activity was recorded, while concentrations of their haemoglobin and Packed Cell Volume (PCV) reduced. The obtained results from these biomarkers were confirmed by the histopathological sections of the animal organs.
Citation | Reginald C. Ohiri; Eugene N. Onyeike; Augustine A. Uwakwe (2021). Toxicological Indices of Wistar Rats Fed Formulated Chaw of Telfairia occidentalis Planted on Crude Oil Contaminated and Remediated Soil. Agriculture and Food Sciences Research, 8(2): 36-43.

History:

Received: 10 September 2021

Revised: 2 December 2021

Accepted: 20 December 2021

Published: 14 January 2022

Licensed: This work is licensed under a Creative Commons

Attribution 3.0 License $(\mathrm{cc})$ EY

Publisher: Asian Online Journal Publishing Group

\section{Contents}

1. Introduction

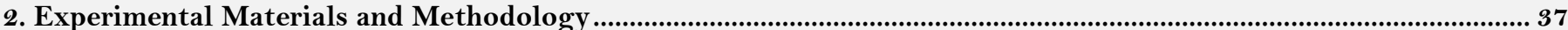

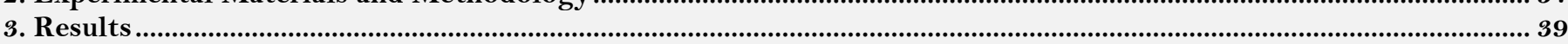

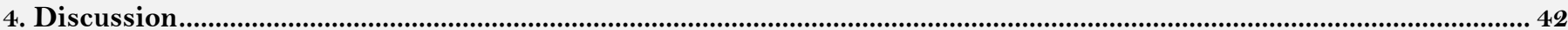

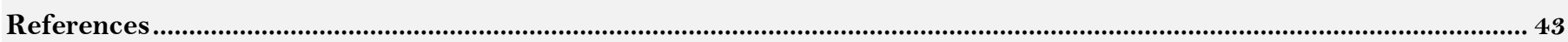
Funding: This study received no specific financial support.

Competing Interests: The authors declare that they have no conflict of interests.

Transparency: The authors confirm that the manuscript is an honest, accurate, and transparent account of the study was reported; that no vital features of the study have been omitted; and that any discrepancies from the as planned have been explained.

Ethical: This study follows all ethical practices during writing. 


\section{Contribution of this paper to the literature}

This study contributes to existing literature by confirming that accumulated hydrocarbons and heavy metals in plants (vegetables) will cause destruction of cellular architecture in animals upon ingestion, thereby causing impairments in cellular activities.

\section{Introduction}

One established mechanism by which plants decontaminate oil contaminated soil is by direct absorption of the contaminant hydrocarbons and subsequent accumulation of same in specific plant tissues and organelles. The Casparian strip has remain a structural foundation for uptake of substances through the plant roots. This system blocks the possible absorption of soil solution via the plant root to the xylem [1]. Substances inside root cells are moved to the aerial parts of the plant. Foliar uptake has been the most important pathway for petroleum hydrocarbon uptake [2]. Other factors that influence hydrocarbon uptake aside from foliar uptake include aqueous concentration of petroleum hydrocarbon, uptake/exposure period, plants bio-physical and chemical compositions. Kipopoulou, et al. [3], reported solubility, octanol-water and octanol-air partition coefficients and vapour pressure as good predictors of petroleum hydrocarbon accumulation in leaf tissues of vegetables. T. occidentalis is a commonly produced crop in the Niger delta area of Nigeria. This region has recorded exorbitant crude oil pollution since the advent of petroleum exploration in the area and there is a continuous annual elevation of its contaminant load in concord with industrial surge and energy uptake thus necessitating the need for environmental remediation. After remediation of petroleum hydrocarbon pollution within and agricultural area, cultivation and growing of plants are carried out without consideration to hydrocarbon recalcitrance in the post remediated soil. These post remediated hydrocarbons may be absorbed, accumulated and a possible high or low potent toxicant can be generated as an intermediary metabolite and stored in specific plant cells. The photosynthetic activities in leafy vegetable (which T. occidentalis is amongst them) enriches them with protein, minerals and carbohydrates [4]. Also referred as fluted pumpkin, T. occidentalis Hoof (Family: Cucurbitaceae) has been a vital commercial crop of cultivated within the humid lowland tropical region situated in the Western part of Africa [5]. Because of its nutritional value, T. occidentalis is prominent in the diet of many animals, including humans [4]. This research was geared towards the evaluation of the toxicological indices of wistar rats fed formulated chaw from T. occidentalis planted on crude oil contaminated and remediated soil.

\section{Experimental Materials and Methodology}

\subsection{Source of Materials}

A volume of $30 \mathrm{dm}^{3}$ of bonny light crude oil was gotten from flow station of Shell Petroleum Development Company located at Egbema Local Government area of Imo State, Nigeria. Forty kilograms (40 kg) of poultry manure was obtained from Godvine Poultry Farm located at Obio Akpor Local Government area of Rivers State, Nigeria. Viable T. occidentalis seeds were obtained from Rumuokoro market, also located in Obio Akpor Local Goverment Rivers State, Nigeria. While a total of 36 healthy wistar rats of 9 rats each of adult males and females, wealing males and females were selected from the research animal house of Biochemistry Department, University of Port Harcourt, River State, Nigeria.

\subsection{Source of Reagent Kits}

Alanine and aspartate aminotransferase, alkaline phosphatase, creatinine and urea kits were sourced from Randox laboratories Ltd, Antrim, United Kingdom, while the kit for alpha amylase was from Giesse Diagnostics, Rome, Italy.

\subsection{Description of Research Site.}

The research site was geographically located on longitude $7^{\circ} 10^{\prime \prime} \mathrm{E}$ and latitude $4^{\circ} 40^{\prime \prime} \mathrm{N}$ on the Eneka axis of Eneka-Oyigbo inter-city road in Obio Akpor, Rivers State, Nigeria. Soils of this research site has an approximate $\mathrm{pH}$ of $4.86 \pm 0.12$, it belongs to the ultisols and are made up of humus topsoil with a deep mixture of sandy and clay soil. Pipeline vandalization and oil spillage has not been recorded on the research site and its environs.

\subsection{Pollution and Remediation of Research Site}

A farmland, measuring $18 \mathrm{~m}^{2}$ was divided into three lots (A, B and C) of $4 \mathrm{~m}^{2}$ and $2 \mathrm{~m}$ land space was allowed between each lot. Two lots were polluted and remediated as follows: Lot B $=4 \mathrm{~m}^{2}$ farmland polluted with $40 \mathrm{dm}^{3}$ of bonny light crude oil and remediated with $40 \mathrm{~kg}$ of poultry manure for 16 weeks (bioaugmented soil). Lot $\mathrm{C}=4$ $\mathrm{m}^{2}$ un-remediated farmland polluted with $40 \mathrm{dm}^{3}$ of bonny light crude oil and allowed for 16 weeks (natural attenuated soil), while Lot $\mathrm{A}=$ Unpolluted $4 \mathrm{~m}^{2}$ farmland (control soil).

\subsection{Planting and Growing and Collection of Vegetable Samples}

After 16 weeks of remediation, viable T. occidentalis seeds were planted on the 3 lots and 4 weeks of growth were allowed from their germination date. Vegetable leaves were harvested at the fourth week with a sterilized razor blade, placed into sterilized cellophane bags and closed with plastic bands. Each cellophane bag of harvested samples was marked using a water-resistant marker and subsequently taken to the laboratory within one hour of sample collection for hydrocarbon and heavy metal analysis.

\subsection{Aliphatic and Polycyclic Aromatic Hydrocarbons (PAH) and Heavy Metals}

Concentration of both aliphatic and polycyclic aromatic hydrocarbons were extracted and determined in $T$. occidentalis by the method described by the AOAC [6], while the method described by Jones Jr [7], was used to determine heavy metal concentration in the vegetables. 


\subsection{Formulation of Rat Chaw}

Harvested leaves of $T$. occidentalis were dried and weighed using model AB204 Mettler Toledo weighing balance and a Model 4. Thomas Scientific Wiley's mill was use to grind the vegetable. The ground sample was formed into rat chaw and combined with normal rat feed.

\subsection{Acclimatization, Treatment and Sacrificing of Research Animals}

Four groups of wistar rats made up of 9 rats each (totalling 36 rats) of adult males and females, wealing males and females were subsequently sub-grouped into 3. Both the control and test animals were placed at normal room temperature in steel cages and allowed to acclimatize for a period of 1 week while having free access to normal rat feed and portable drinking water. The sub-groups were placed on rat chaw comprising of equal ratio of normal rat feed and dried $T$. occidentalis from the control, bioaugmented and natural attenuated lots. Using chloroform anaesthesia, the animals were sacrificed at the $28^{\text {th }}$ day and blood sample was obtained via cardiac puncture into ethylene diamine tetracetic acid (EDTA) bottles and taken to the laboratory within one hour for assay of the following toxicological indices: alkaline phosphatase, alanine and aspartate aminotransferases, urea, creatinine, hemoglobin concentration and packed cell volume (PCV). The internal organs (liver, kidney and pancreas) were immediately harvested into a preservative container of $10 \%$ physiological saline and subsequently subjected to histological analyses.

Table-1. Concentration of aliphatic hydrocarbon $\left(\mathrm{mgkg}^{-1}\right)$ in T. occidentalis from crude oil remediated soil.

\begin{tabular}{|c|c|c|c|}
\hline No. of Carbon atoms & Control soil & Bioaugmented soil & Natural attenuated soil \\
\hline $\mathrm{C}_{8}$ & $2.29 \pm 0.05^{\mathrm{a}}$ & $1.30 \pm 0.03^{\mathrm{b}}$ & $5.37 \pm 0.20^{\mathrm{c}}$ \\
\hline $\mathrm{C}_{9}$ & $1.60 \pm 0.03^{\mathrm{ab}}$ & $1.50 \pm 0.03^{\mathrm{ab}}$ & $2.78 \pm 0.04^{\mathrm{c}}$ \\
\hline $\mathrm{C}_{10}$ & $1.04 \pm 0.02^{\mathrm{ac}}$ & $4.43 \pm 0.05^{b}$ & $3.02 \pm 0.03^{\mathrm{ac}}$ \\
\hline $\mathrm{C}_{11}$ & $1.07 \pm 0.07^{\mathrm{a}}$ & $7.56 \pm 0.10^{\mathrm{b}}$ & $2.11 \pm 0.04^{c}$ \\
\hline $\mathrm{C}_{12}$ & $2.70 \pm 0.02^{\mathrm{a}}$ & $1.23 \pm 0.01^{b}$ & $1.56 \pm 0.08^{\mathrm{c}}$ \\
\hline $\mathrm{C}_{13}$ & $2.05 \pm 0.02^{\mathrm{a}}$ & $2.31 \pm 0.06^{\mathrm{b}}$ & $1.02 \pm 0.01^{\mathrm{c}}$ \\
\hline $\mathrm{C}_{14}$ & $1.47 \pm 0.09^{\mathrm{ac}}$ & $8.17 \pm 0.02^{b}$ & $1.43 \pm 0.01^{\mathrm{ac}}$ \\
\hline $\mathrm{C}_{15}$ & $1.66 \pm 0.09^{\mathrm{a}}$ & $1.19 \pm 0.02^{b}$ & $1.02 \pm 0.02^{\mathrm{c}}$ \\
\hline $\mathrm{C}_{16}$ & $1.03 \pm 0.01^{\mathrm{a}}$ & $3.12 \pm 0.01^{b}$ & $7.90 \pm 0.18^{\mathrm{c}}$ \\
\hline $\mathrm{C}_{17}$ & $1.02 \pm 0.05^{\mathrm{a}}$ & $3.02 \pm 0.02^{b}$ & $5.83 \pm 0.24^{c}$ \\
\hline Pristane & $1.17 \pm 0.06^{\mathrm{a}}$ & $6.65 \pm 0.21^{b}$ & $5.08 \pm 0.05^{\mathrm{c}}$ \\
\hline $\mathrm{C}_{18}$ & $1.32 \pm 0.03^{\mathrm{a}}$ & $2.08 \pm 0.14^{b}$ & $6.95 \pm 0.28^{c}$ \\
\hline Phytane & $1.61 \pm 0.01^{\mathrm{a}}$ & $1.11 \pm 0.02^{b}$ & $4.14 \pm 0.02^{c}$ \\
\hline $\mathrm{C}_{19}$ & $1.90 \pm 0.05^{\mathrm{a}}$ & $4.18 \pm 0.02^{b}$ & $6.83 \pm 0.06^{c}$ \\
\hline $\mathrm{C}_{20}$ & $8.56 \pm 0.06^{\mathrm{a}}$ & $1.65 \pm 0.02^{\mathrm{b}}$ & $6.17 \pm 0.06^{c}$ \\
\hline $\mathrm{C}_{21}$ & $1.53 \pm 0.08^{\mathrm{a}}$ & $9.03 \pm 0.09^{b}$ & $1.86 \pm 0.09^{c}$ \\
\hline $\mathrm{C}_{22}$ & $2.76 \pm 0.07^{\mathrm{a}}$ & $2.46 \pm 0.10^{b}$ & $1.19 \pm 0.04^{\mathrm{c}}$ \\
\hline $\mathrm{C}_{23}$ & $5.49 \pm 0.04^{\mathrm{a}}$ & $3.07 \pm 0.14^{b}$ & $1.80 \pm 0.02^{\mathrm{c}}$ \\
\hline $\mathrm{C}_{24}$ & $6.83 \pm 0.08^{\mathrm{a}}$ & $2.30 \pm 0.03^{b}$ & $1.61 \pm 0.02^{\mathrm{c}}$ \\
\hline $\mathrm{C}_{25}$ & $3.14 \pm 0.06^{\mathrm{a}}$ & $6.36 \pm 0.07^{\mathrm{b}}$ & $1.04 \pm 0.00^{\mathrm{c}}$ \\
\hline $\mathrm{C}_{26}$ & $6.49 \pm 0.03^{\mathrm{a}}$ & $2.02 \pm 0.02^{b}$ & $7.46 \pm 0.09^{c}$ \\
\hline $\mathrm{C}_{27}$ & $7.80 \pm 0.09^{\mathrm{a}}$ & $9.58 \pm 0.09^{b}$ & $9.10 \pm 0.20^{\mathrm{c}}$ \\
\hline $\mathrm{C}_{28}$ & $1.22 \pm 0.01^{\mathrm{a}}$ & $1.66 \pm 0.07^{b c}$ & $1.83 \pm 0.11^{b c}$ \\
\hline $\mathrm{C}_{29}$ & $1.59 \pm 0.05^{\mathrm{a}}$ & $6.17 \pm 0.06^{\mathrm{b}}$ & $7.35 \pm 0.15^{\mathrm{c}}$ \\
\hline $\mathrm{C}_{30}$ & $1.17 \pm 0.03^{\mathrm{a}}$ & $3.89 \pm 0.03^{b}$ & $5.11 \pm 0.01^{\mathrm{c}}$ \\
\hline $\mathrm{C}_{31}$ & $1.54 \pm 0.10^{\mathrm{a}}$ & $3.87 \pm 0.05^{\mathrm{b}}$ & $5.46 \pm 0.08^{\mathrm{c}}$ \\
\hline $\mathrm{C}_{32}$ & $6.09 \pm 0.03^{\mathrm{a}}$ & $9.03 \pm 0.05^{b}$ & $7.03 \pm 0.01^{\mathrm{c}}$ \\
\hline $\mathrm{C}_{33}$ & $5.23 \pm 0.07^{\mathrm{a}}$ & $1.47 \pm 0.05^{\mathrm{b}}$ & $3.11 \pm 0.01^{\mathrm{c}}$ \\
\hline $\mathrm{C}_{34}$ & $2.51 \pm 0.02^{\mathrm{a}}$ & $5.08 \pm 0.03^{b}$ & $6.44 \pm 0.07^{c}$ \\
\hline $\mathrm{C}_{35}$ & $4.18 \pm 0.10^{\mathrm{a}}$ & $3.55 \pm 0.08^{b}$ & $7.60 \pm 0.02^{c}$ \\
\hline $\mathrm{C}_{36}$ & $2.82 \pm 0.44^{\mathrm{a}}$ & $4.03 \pm 0.00^{b}$ & $9.34 \pm 0.03^{c}$ \\
\hline $\mathrm{C}_{37}$ & $9.08 \pm 0.04^{\mathrm{a}}$ & $5.97 \pm 0.21^{b}$ & $2.09 \pm 0.01^{\mathrm{c}}$ \\
\hline $\mathrm{C}_{38}$ & $2.26 \pm 0.08^{\mathrm{ab}}$ & $2.19 \pm 0.04^{\mathrm{ab}}$ & $7.18 \pm 0.02^{\mathrm{c}}$ \\
\hline $\mathrm{C}_{39}$ & $6.37 \pm 0.14^{\mathrm{a}}$ & $8.79 \pm 0.04^{b}$ & $5.84 \pm 0.09^{\mathrm{c}}$ \\
\hline $\mathrm{C}_{40}$ & $2.55 \pm 0.08^{\mathrm{a}}$ & $6.43 \pm 0.05^{\mathrm{b}}$ & $1.58 \pm 0.07^{\mathrm{c}}$ \\
\hline
\end{tabular}

\section{Note:}

Data presented are mean values \pm standard deviations of three observations.

Superscript "a" indicates statistical significance at $\mathrm{P}<0.05$ when control lot is compared to the other lots.

Superscript "b" indicates statistical significance at $\mathrm{P}<0.05$ when bioaugmented lot is compared to the other lots.

Superscript " $\mathrm{c}$ " indicates statistical significance at $\mathrm{P}<0.05$ when natural attenuated lot is compared to the other lots.

Superscript "ab" indicates statistical insignificance at $\mathrm{P}<0.05$ when control and bioaugmented lots are compared to each other, But statistically significant when both are compared to the natural attenuated lot.

Superscript "ac" indicates statistical insignificance at $\mathrm{P}<0.05$ when control and natural attenuated lots are compared to each other,

But statistically significant when both are compared to the bioaugmented lot.Superscript "bc" indicates statistical insignificance at $\mathrm{P}<0.05$ when bioaugmented and natural attenuated lots are compared to each other, but statistically significant when both are compared to the control lot.

\subsection{Assay of Some Toxicological Parameters of Wistar Rats Fed with Vegetables from Bioremediated Soil.}

The diacetyl monoxine method (DAM), described by Marsh, et al. [8], was used to assay for serum Urea concentration while the Alkaline picric acid procedure described by Slot [9], was used to determine the concentration of Creatinine. The 2-chloro-p-nitrophenyl- $\alpha$-D-maltotrioside (CNPG3) kinetic procedure described by Hohenwallner, et al. [10] and the assay procedure of Kind and King [11], were used to determine Amylase and Alkaline phosphatase activities respectively, while the colorimetric end-point procedure of described by Reitman and Frankel [12] was used to determine both Aspartate and Alanine aminotransferases. Corash [13], procedure was used the haematocrit (PCV) volume and haemoglobin concentration, while histopathological assay of animal 
tissues was done using haematoxylin and eosin procedure described by Conn [14] and modified by Kumar and Gill [15].

\subsection{Statistical Analysis/Test of Significance}

The mean \pm standard deviations and One Way Analysis of Variance were calculated electronically for each group of observations using International Business Machine (IBM) Statistical Program for Social Sciences (SPSS) 19 software at $95 \%$ confidence level, while Duncan [16], post hoc multiple range test was used to test for significance.

\section{Results}

Hydrocarbon and heavy metal content of vegetable samples. The mean values of both aliphatic hydrocarbons, polycyclic aromatic hydrocarbons and heavy metals obtained in T. occidentalis grown on the 3 lots (control, bioaugmented and natural attenuated) are presented in Tables 1-3. A significant increase at $\mathrm{P}<0.05$ in aliphatic hydrocarbon content was observed in T. occidentalis harvested from natural attenuated and bioaugmented soils aside $\mathrm{C}_{20}, \mathrm{C}_{24}$ and $\mathrm{C}_{37}$ where higher concentrations were recorded in the control vegetables than in the remediated soils Polycyclic aromatic hydrocarbon content of $T$. occidentalis increased in vegetables harvested from both natural attenuated and bioaugmented aside acenaphthene, phenanthrene and indeno(1,2,3-cd)pyrene. Higher concentration of acenaphthene was recorded in T. occidentalis from the control lot than in those from both the natural attenuated and bioaugmented soil. Phenanthrene concentrations for those grown on the control and natural attenuated soil were equal with a value of $2.67 \mathrm{mgkg}^{-1}$, while Phenanthrene concentration in vegetable samples from the bioaugmented soil increased significantly. A significantly lower concentration of Indeno(1,2,3-cd)pyrene was observed at $\mathrm{P}<0.05$ in vegetables harvested from the natural attenuated soil than in those from the control soil. A significantly high concentration at $\mathrm{P}<0.05$ of heavy metals were observed in vegetables from the bioaugmented soil as compared to those from both the control and the natural attenuated lots.

\begin{tabular}{|c|c|c|c|}
\hline Polycyclic aromatic hydrocarbon & Control soil & Bioaugmented soil & Natural attenuated soil \\
\hline Naphthalene & $0.55 \pm 0.07^{\mathrm{a}}$ & $3.98 \pm 0.05^{\mathrm{b}}$ & $2.95 \pm 0.04^{\mathrm{c}}$ \\
\hline Acenaphthylene & $1.58 \pm 0.03^{\mathrm{a}}$ & $2.87 \pm 0.01^{\mathrm{b}}$ & $3.61 \pm 0.01^{\mathrm{c}}$ \\
\hline Acenaphthene & $1.87 \pm 0.05^{\mathrm{ab}}$ & $1.73 \pm 0.03^{\mathrm{ab}}$ & $1.57 \pm 0.08^{\mathrm{c}}$ \\
\hline Fluorene & $2.60 \pm 0.05^{\mathrm{a}}$ & $4.44 \pm 0.04^{b}$ & $5.34 \pm 0.01^{\mathrm{c}}$ \\
\hline Phenanthrene & $2.67 \pm 0.01^{\mathrm{ac}}$ & $3.02 \pm 0.03^{b}$ & $2.67 \pm 0.04^{\mathrm{ac}}$ \\
\hline Anthracene & $2.33 \pm 0.01^{\mathrm{a}}$ & $3.56 \pm 0.04^{b}$ & $3.80 \pm 0.01^{\mathrm{c}}$ \\
\hline Fluoranthene & $1.57 \pm 0.01^{\mathrm{a}}$ & $7.03 \pm 0.02^{b}$ & $9.18 \pm 0.05^{\mathrm{c}}$ \\
\hline Pyrene & $\mathrm{NP}$ & $\mathrm{NP}$ & NP \\
\hline $\operatorname{Benzo}(\alpha)$ anthracene & $1.77 \pm 0.08^{\mathrm{a}}$ & $7.24 \pm 0.07^{\mathrm{b}}$ & $6.09 \pm 0.04^{c}$ \\
\hline Chrysene & $0.07 \pm 0.01^{\mathrm{a}}$ & $2.52 \pm 0.11^{b}$ & $2.73 \pm 0.02^{\mathrm{c}}$ \\
\hline Benzo $(\beta)$ fluoranthene & $1.69 \pm 0.06^{\mathrm{a}}$ & $1.85 \pm 0.08^{b}$ & $2.68 \pm 0.04^{c}$ \\
\hline Benzo(k)fluoranthene & $\mathrm{NP}$ & $\mathrm{NP}$ & $\mathrm{NP}$ \\
\hline $\operatorname{Benzo}(\alpha)$ pyrene & $0.53 \pm 0.02^{\mathrm{a}}$ & $2.98 \pm 0.10^{\mathrm{bc}}$ & $3.02 \pm 0.00^{b c}$ \\
\hline Indeno $(1,2,3)$ pyrene & $2.27 \pm 0.01^{\mathrm{a}}$ & $3.28 \pm 0.03^{b}$ & $4.15 \pm 0.03^{\mathrm{c}}$ \\
\hline Indeno(1,2,3-cd)pyrene & $3.47 \pm 0.07^{\mathrm{a}}$ & $6.75 \pm 0.06^{\mathrm{b}}$ & $0.43 \pm 0.03^{c}$ \\
\hline Dibenz(a,h)anthracene & $1.66 \pm 0.00^{\mathrm{a}}$ & $2.58 \pm 0.04^{b}$ & $1.89 \pm 0.05^{\mathrm{c}}$ \\
\hline Benzo(g,h,i)perylene & $\mathrm{NP}$ & $\mathrm{NP}$ & $\mathrm{NP}$ \\
\hline
\end{tabular}

\section{Note:}

Presented are mean values \pm standard deviations of three observations. NP $=$ Not Present.

Superscript "a" indicates statistical significance at $\mathrm{P}<0.05$ when control lot is compared to the other lots.

Superscript "b" indicates statistical significance at $\mathrm{P}<0.05$ when bioaugmented lot is compared to the other lots.

Superscript "b" indicates statistical significance at $\mathrm{P}<0.05$ when bioaugmented lot is compared to the other lots.
Superscript "c" indicates statistical significance at $\mathrm{P}<0.05$ when natural attenuated lot is compared to the other lots.

Superscript "ab" indicates statistical insignificance at $\mathrm{P}<0.05$ when control and bioaugmented lots are compared to each other, But statistically significant when both are compared to the natural attenuated lot.

Superscript "ac" indicates statistical insignificance at $\mathrm{P}<0.05$ when control and natural attenuated lots are compared to each other But statistically significant when both are compared to the bioaugmented lot.

Superscript "bc" indicates statistical insignificance at $\mathrm{P}<0.05$ when bioaugmented and natural attenuated lots are compared to each other,

But statistically significant when both are compared to the control lot.

\begin{tabular}{c|c|c|c}
\multicolumn{4}{c}{ Table-3. Heavy metal concentrations $(\mathrm{g} / 100 \mathrm{~g})$ of $T$. occidentalis from crude oil bioremediated soil. } \\
\hline Heavy metal & Control soil & Bioaugmented soil & Natural attenuated soil \\
\hline Copper & $0.01 \pm 0.0^{\mathrm{ac}}$ & $0.03 \pm 0.00^{\mathrm{b}}$ & $0.01 \pm 0.00^{\mathrm{ac}}$ \\
\hline Lead & $\mathrm{NP}$ & $0.10 \pm 0.02^{\mathrm{b}}$ & $0.08 \pm 0.00^{\mathrm{c}}$ \\
\hline Chromium & $\mathrm{NP}$ & $0.06 \pm 0.00^{\mathrm{b}}$ & $\mathrm{NP}$ \\
\hline Cadmium & $\mathrm{NP}$ & $0.03 \pm 0.00^{\mathrm{b}}$ & $0.01 \pm 0.00^{\mathrm{c}}$ \\
\hline Arsenic & $\mathrm{NP}$ & $\mathrm{NP}$ & $\mathrm{NP}$ \\
\hline
\end{tabular}

Note:
Data presented are mean values \pm standard deviations of three observations. $\mathrm{NP}=$ Not Present

Superscript "a" indicates statistical significance at $\mathrm{P}<0.05$ when control lot is compared to the other lots.

Superscript "b" indicates statistical significance at $\mathrm{P}<0.05$ when bioaugmented lot is compared to the other lots.

Superscript "c" indicates statistical significance at $\mathrm{P}<0.05$ when natural attenuated lot is compared to the

other lots.
Superscript "ac" indicates statistical insignificance at $\mathrm{P}<0.05$ when control and natural attenuated lots are compared to each other,

But statistically significant when both are compared to the bioaugmented lot. 
3.1. Indicators of Tissue Damage and Haematological Parameters

Table-4. Serum urea and creatinine concentration (mgdl-1) of rats fed T. occidentalis from crude oil bioremediated soil.

\begin{tabular}{l|c|c|c}
\hline & Control soil & Bioaugmented soil & Natural attenuated soil \\
\hline \multicolumn{3}{|c|}{ Urea } \\
\hline Weanling male & $30.19 \pm 0.13^{\mathrm{a}}$ & $54.35 \pm 0.51^{\mathrm{bc}}$ & $54.32 \pm 0.11^{\mathrm{bc}}$ \\
\hline Weanling female & $31.40 \pm 1.07^{\mathrm{a}}$ & $56.80 \pm 0.20^{\mathrm{bc}}$ & $55.50 \pm 0.36^{\mathrm{bc}}$ \\
\hline Adult male & $33.62 \pm 0.18^{\mathrm{a}}$ & $52.00 \pm 0.63^{\mathrm{b}}$ & $58.92 \pm 0.47^{\mathrm{c}}$ \\
\hline Adult female & $32.93 \pm 0.19^{\mathrm{a}}$ & $58.61 \pm 0.30^{\mathrm{bc}}$ & $58.66 \pm 0.43^{\mathrm{bc}}$ \\
\hline \multicolumn{3}{|c|}{ Creatinine } & $1.82 \pm 0.09^{\mathrm{bc}}$ \\
\hline Weanling male & $1.19 \pm 0.02^{\mathrm{a}}$ & $1.73 \pm 0.08^{\mathrm{bc}}$ & $1.72 \pm 0.02^{\mathrm{bc}}$ \\
\hline Weanling female & $1.11 \pm 0.02^{\mathrm{a}}$ & $1.79 \pm 0.07^{\mathrm{bc}}$ & $1.71 \pm 0.05^{\mathrm{bc}}$ \\
\hline Adult male & $0.94 \pm 0.03^{\mathrm{a}}$ & $1.66 \pm 0.03^{\mathrm{bc}}$ & $\underline{0.08^{\mathrm{bc}}}$ \\
\hline Adult female & $0.80 \pm 0.01^{\mathrm{a}}$ & $1.90 \pm 0.03^{\mathrm{bc}}$ &
\end{tabular}

Note:

Data presented are mean values \pm standard deviations of three observations.

Superscript " "" indicates statistical significance at $\mathrm{P}<0.05$ when control lot is compared to the other lots.

Superscript " $\mathrm{b}$ " indicates statistical significance at $\mathrm{P}<0.05$ when bioaugmented lot is compared to the other lots.

Superscript " $\mathrm{c}$ " indicates statistical significance at $\mathrm{P}<0.05$ when natural attenuated lot is compared to the other lots.

Superscript "bc" indicates statistical insignificance at $\mathrm{P}<0.05$ when bioaugmented and natural attenuated lots are compared to each other,

But statistically significant when both are compared to the control lot.

Table-5. Serum enzymes concentration (I.Ul-1) of rats fed T. occidentalis grown on crude oil bioremediated soil.

\begin{tabular}{|c|c|c|c|}
\hline & Control soil & Bioaugmented soil & Natural attenuated soil \\
\hline \multicolumn{4}{|c|}{ Alkaline phosphatase } \\
\hline Weanling male & $284.00 \pm 5.10^{\mathrm{a}}$ & $486.90 \pm 10.40^{\mathrm{b}}$ & $357.00 \pm 3.20^{\mathrm{c}}$ \\
\hline Weanling female & $268.22 \pm 1.49^{\mathrm{a}}$ & $479.81 \pm 0.40^{\mathrm{b}}$ & $344.02 \pm 5.63^{\mathrm{c}}$ \\
\hline Adult male & $243.71 \pm 1.91^{\mathrm{a}}$ & $346.20 \pm 1.50^{\mathrm{b}}$ & $318.07 \pm 1.20^{\mathrm{c}}$ \\
\hline Adult female & $226.53 \pm 1.72^{\mathrm{a}}$ & $359.70 \pm 0.84^{\mathrm{b}}$ & $304.39 \pm 0.75^{\mathrm{c}}$ \\
\hline \multicolumn{4}{|c|}{ Alanine aminotransferase } \\
\hline Weanling male & $18.00 \pm 0.68^{\mathrm{a}}$ & $61.28 \pm 0.24^{\mathrm{b}}$ & $67.42+2.02^{\mathrm{c}}$ \\
\hline Weanling female & $20.60 \pm 0.41^{\mathrm{a}}$ & $57.63 \pm 0.53^{\mathrm{b}}$ & $64.19 \pm 1.15^{\mathrm{c}}$ \\
\hline Adult male & $25.20 \pm 0.84^{\mathrm{a}}$ & $48.45 \pm 1.31^{\mathrm{b}}$ & $61.59 \pm 0.75^{\mathrm{c}}$ \\
\hline Adult female & $24.00 \pm 0.63^{\mathrm{a}}$ & $41.05 \pm 0.43^{\mathrm{b}}$ & $60.42 \pm 0.40^{\mathrm{c}}$ \\
\hline \multicolumn{4}{|c|}{ Aspartate aminotransferase } \\
\hline Weanling male & $19.00 \pm 0.28^{\mathrm{a}}$ & $61.00 \pm 1.61^{\mathrm{bc}}$ & $63.05 \pm 0.76^{\mathrm{bc}}$ \\
\hline Weanling female & $19.22+0.80^{\mathrm{a}}$ & $57.37 \pm 033^{\mathrm{bc}}$ & $58.03 \pm 0.07^{\mathrm{bc}}$ \\
\hline Adult male & $32.81 \pm 1.14^{\mathrm{a}}$ & $57.60 \pm 0.02^{\mathrm{b}}$ & $62.40 \pm 0.60^{\mathrm{c}}$ \\
\hline Adult female & $29.70 \pm 0.67^{\mathrm{a}}$ & $55.00 \pm 1.02^{\mathrm{b}}$ & $63.81 \pm 0.91^{\mathrm{c}}$ \\
\hline \multicolumn{4}{|c|}{ Amylase } \\
\hline Weanling male & $27.66+0.46^{\mathrm{a}}$ & $29.49+0.54^{\mathrm{bc}}$ & $29.07+0.23 b c$ \\
\hline Weanling female & $26.85+0.34^{\mathrm{a}}$ & $31.09+0.61^{b c}$ & $31.47+1.03^{\mathrm{bc}}$ \\
\hline Adult male & $32.83+0.36^{\mathrm{a}}$ & $38.18+0.86^{\mathrm{b}}$ & $35.17+0.82^{\mathrm{c}}$ \\
\hline Adult female & $33.51+0.47^{\mathrm{a}}$ & $36.89+0.50^{\text {bc }}$ & $36.73+0.37^{\mathrm{bc}}$ \\
\hline
\end{tabular}

Note:

Data presented are mean values + standard deviations of three observations.

Superscript "a" indicates statistical significance at $\mathrm{P}<0.05$ when control lot is compared to the other lots.

Superscript "b" indicates statistical significance at $\mathrm{P}<0.05$ when bioaugmented lot is compared to the other lots.

Superscript "c" indicates statistical significance at $\mathrm{P}<0.05$ when natural attenuated lot is compared to the other lots.

Superscript "bc" indicates statistical insignificance at $\mathrm{P}<0.05$ when bioaugmented and natural attenuated lots are compared to each other,

But statistically significant when both are compared to the control lot

Table-6. Packed Cell Volume (PCV) (\%) and Haemoglobin concentration of rats fed T. occidentalis from crude oil bioremediated soil

\begin{tabular}{|c|c|c|c|}
\hline & Control soil & Bioaugmented soil & Natural attenuated soil \\
\hline \multicolumn{4}{|c|}{ Packed Cell Volume (\%) } \\
\hline Weanling male & $31.35+1.33^{\mathrm{a}}$ & $24.35 \pm 1.53^{\mathrm{bc}}$ & $24.00 \pm 1.01^{\mathrm{bc}}$ \\
\hline Weanling female & $31.67 \pm 0.58^{\mathrm{a}}$ & $25.00 \pm 2.04^{\mathrm{bc}}$ & $21.68 \pm 0.59^{\mathrm{bc}}$ \\
\hline Adult male & $34.34 \pm 2.52^{\mathrm{a}}$ & $25.00 \pm 3.61^{\mathrm{bc}}$ & $25.33 \pm 2.52^{\mathrm{bc}}$ \\
\hline Adult female & $36.33 \pm 0.57^{\mathrm{a}}$ & $26.36 \pm 1.16^{\mathrm{b}}$ & $23.67 \pm 0.56^{\mathrm{c}}$ \\
\hline \multicolumn{4}{|c|}{ Haemoglobin $\left(\mathrm{gdl}^{-1}\right)$} \\
\hline Weanling male & $10.44 \pm 0.51^{\mathrm{a}}$ & $7.97 \pm 0.62^{b c}$ & $8.20 \pm 0.48^{\mathrm{bc}}$ \\
\hline Weanling female & $10.56 \pm 0.20^{\mathrm{a}}$ & $8.33 \pm 0.67^{\mathrm{bc}}$ & $7.22 \pm 0.19^{\mathrm{bc}}$ \\
\hline Adult male & $11.44 \pm 0.84^{\mathrm{a}}$ & $8.34 \pm 1.21^{\mathrm{bc}}$ & $8.44 \pm 0.84^{\mathrm{bc}}$ \\
\hline Adult female & $12.11 \pm 0.19^{\mathrm{a}}$ & $8.78 \pm 0.39^{b}$ & $7.89 \pm 0.20^{\mathrm{c}}$ \\
\hline
\end{tabular}

Note:

Data presented are mean values \pm standard deviations of three observations

Superscript "a" indicates statistical significance at $\mathrm{P}<0.05$ when control lot is compared to the other lots.

Superscript "b" indicates statistical significance at $\mathrm{P}<0.05$ when bioaugmented lot is compared to the other lots.

Superscript "c" indicates statistical significance at $\mathrm{P}<0.05$ when natural attenuated lot is compared to the other lots.

Superscript "bc" indicates statistical insignificance at $\mathrm{P}<0.05$ when bioaugmented and natural attenuated lots are compared to each other,

But statistically significant when both are compared to the control lot.

Tables 4- 6 shows mean concentrations of Urea, Creatinine, Amylase, Alkaline phosphatase (ALP), Aspartate and Alanine aminotransferases, Packed cell volume and Hemoglobin concentrations of rats fed with $50 \%$ formulated chaw from the research lots. A significant increase at $\mathrm{P}<0.05$ were observed in all the maker compounds (urea, creatinine, amylase, alkaline phosphatase, alanine and aspartate aminotransferases) of rats fed $50 \%$ chaw from the bioaugmented and natural attenuated lots, while a more pronounced decrease in Packed cell volume and Hemoglobin were recorded in rats fed $50 \%$ chaw formulated from vegetables gotten from the 
bioaugmented lot than in those fed T. occidentalis chaw formulated from vegetables gotten from the natural attenuated lot.

\subsection{Sections of Rat Organs Fed with T. Occidentalis from Crude Oil Bioremediated Lots}

Figure 1 shows histological scores of sections of rat organs fed with T. occidentalis formulated chaw from the crude oil bioremediated lots. The observed lesions in the organ sections were highest in the kidney, liver and pancreas of animals fed T. occidentalis from natural attenuated and bioaugmented lots, while organ sections of those fed $T$. occidentalis from the control lot had healthier sections with non-significant degrees of lesions.

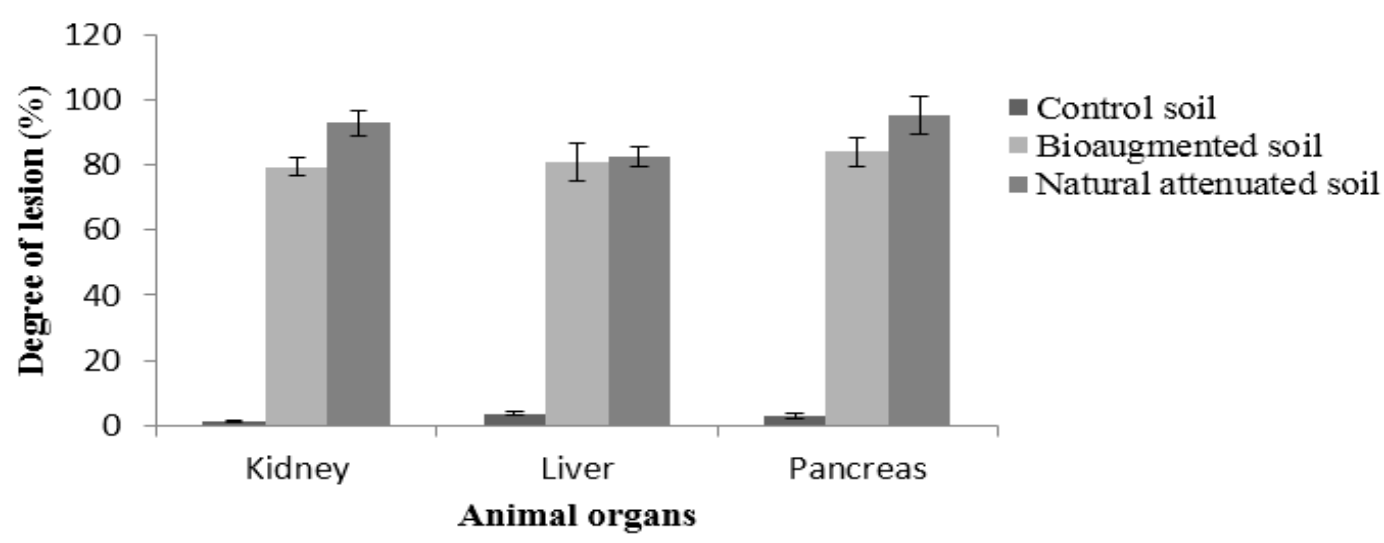

Values are means \pm standard deviations of three determination.

Figure-1. Histological scores of sections of rat organs fed T. occidentalis from crude oil bioremediated soil.

Sections of rat organs fed T. occidentalis from on crude oil bioremediated lots are presented in Figures $2-4$. Animals fed T. occidentalis from the control lot had healthy kidneys with perfect structures of glomeruli and unaltered tissue architecture Figure 2a; while those fed $T$. occidentalis from both bioaugmented and natural attenuated lots had unhealthy distorted organs with shrunken glomeruli and multicystic spaces Figure $2 \mathrm{~b}-2 \mathrm{c}$.

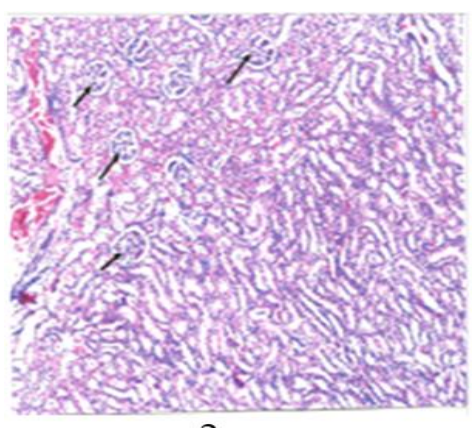

$2 \mathrm{a}$

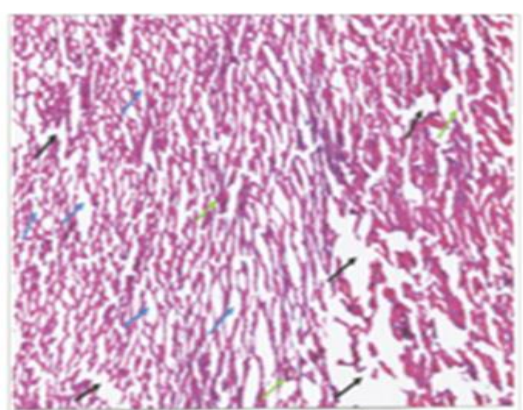

$2 \mathrm{~b}$

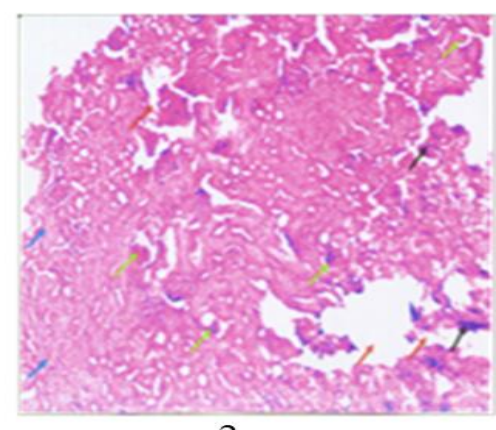

$2 \mathrm{c}$

(a). Representative section of the kidney of rat fed T. occidentalis from control lot. [Glomeruli perfectly in order (Black pointers)]. (b). Representative section of the kidney of rat fed with T. occidentalis from bioaugmented lot. [Observations: Distorted tissue architecture (black pointers), multicystic spaces (blue pointers), glomeruli shrunken (green pointers)]. (c.). Representative section of the kidney of rat fed T. occidentalis from natural attenuated lot. [Mild oedema (black pointers), tissue stromal proliferation with silt-like channel (blue pointers), glomeruli shrunken (green pointers), distorted tissue architecture (red pointers)].

Rats fed T. occidentalis from the control lot had healthy liver sections with well identified hepatocytes and pronounced central vein Figure 3a. The presence of slit like channels and proliferation of tissue was noticed around the central veins of the sections of those fed T. occidentalis from the bioaugmented lot Figure $3 \mathrm{~b}$, while those fed $T$. occidentalis from the natural attenuated lot had proliferated tissue, enlarged central vein and an altered tissue architecture Figure 3c.

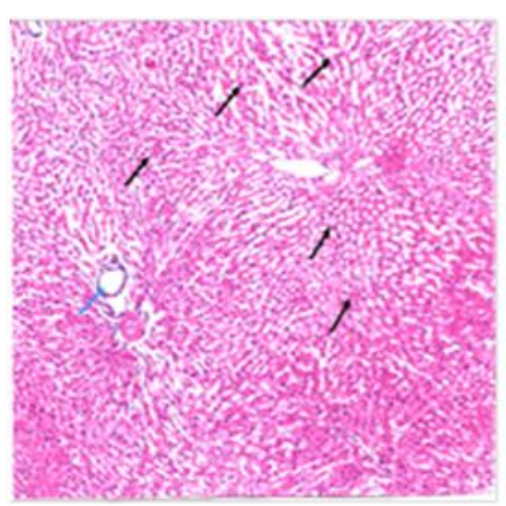

$\begin{array}{ccc}3 \mathrm{a} & 3 \mathrm{~b} & 3 \mathrm{c} \\ \text { Figure-3. Histology Sections of Rat Liver Fed T. Occidentalis from Crude Oil Polluted and Remediated Soil. }\end{array}$

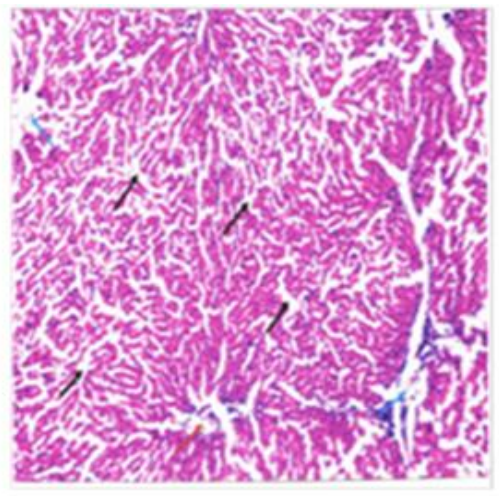

$\begin{array}{ccc}3 \mathrm{a} & 3 \mathrm{~b} & 3 \mathrm{c} \\ \text { Figure-3. Histology Sections of Rat Liver Fed T. Occidentalis from Crude Oil Polluted and Remediated Soil. }\end{array}$

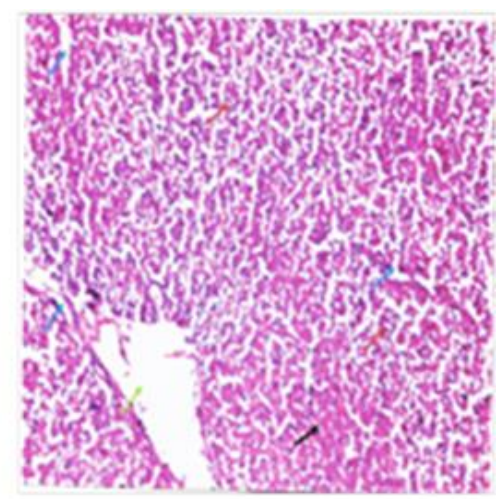

$\begin{array}{ccc}3 \mathrm{a} & 3 \mathrm{~b} & 3 \mathrm{c} \\ \text { Figure-3. Histology Sections of Rat Liver Fed T. Occidentalis from Crude Oil Polluted and Remediated Soil. }\end{array}$

(a). Representative section of the liver of rat fed T. occidentalis from control lot. [Hepatocytes clearly identified (Black pointers), central vein perfectly in order (Blue pointers)]. (b). Representative section of the liver of rat fed $T$. 
occidentalis from bioaugmented lot. [Presence of slit like channels (Black pointers), tissue proliferation (Blue pointers), central vein blocked (Red pointers)]. (c) Representative section of the liver of rat fed T. occidentalis from natural attenuated lot. [Tissue stromal proliferation (black pointers), distorted tissue architecture (blue pointers), hepatocytes not clearly identified (red pointers), central vein grossly enlarged (green pointers)].

Pancreas sections of rats fed T. occidentalis from control lot revealed a healthy, undistorted pancreas Figure 4a, while those fed $T$. occidentalis from both the bioaugmented and natural attenuated lot had scanty stroma, altered tissue architecture and mild oedema Figure $4 \mathrm{~b}$ and $4 \mathrm{c}$.

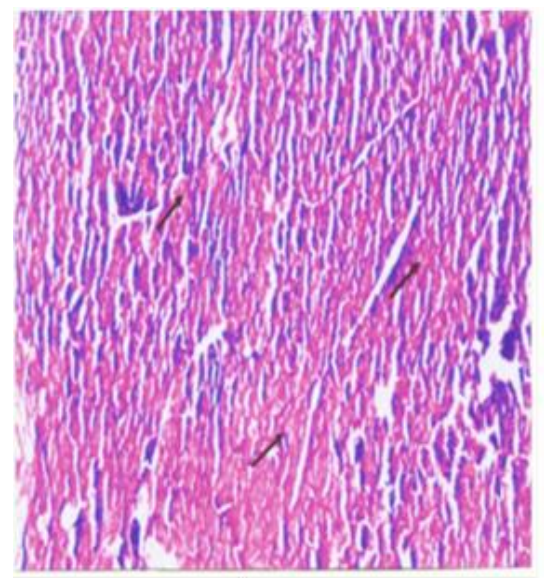

$4 \mathrm{a}$

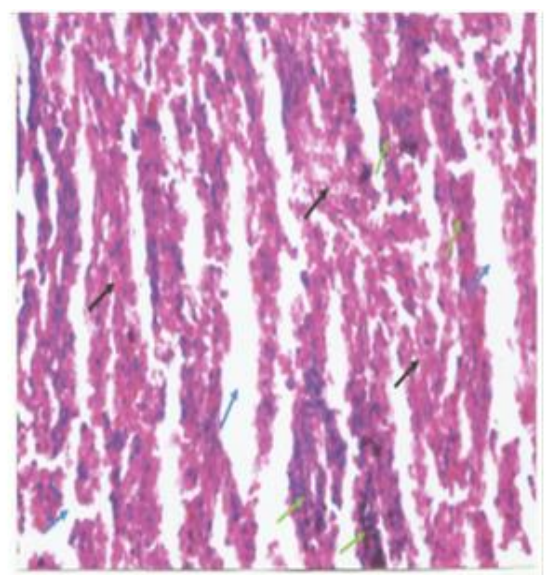

$4 \mathrm{~b}$

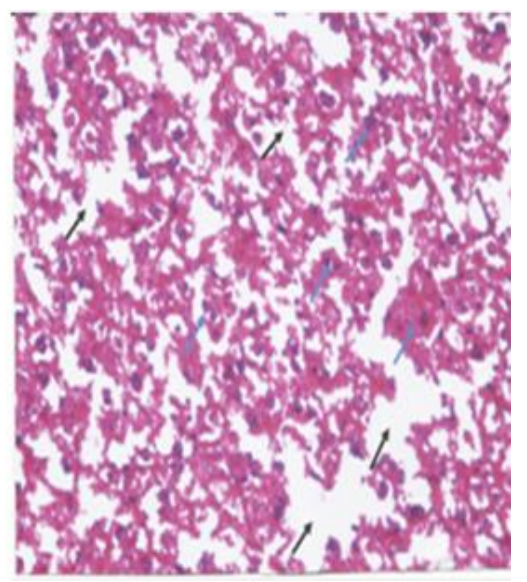

$4 \mathrm{c}$

(a). Representative section of Pancreas of rat fed T. occidentalis from control lot. [Secretory cells perfectly in order (Black pointers)]. (b). Representative section of Pancreas of rat fed T. occidentalis from bioaugmented lot. [Scanty stroma (Black pointers), stromal abrasions (Blue pointers), mild oedema (Green pointers)]. (c). Representative section of Pancreas of rat fed T. occidentalis from natural attenuated lot. [Scanty stroma (Black pointers), pancreatic acini not clearly seen (Blue pointers)].

\section{Discussion}

The accumulation of petroleum hydrocarbons and heavy metals in the leaves $T$. occidentalis of both the bioaugmented and natural attenuated lots can be linked to partial degradation and recalcitrant hydrocarbons present in the remediated lots (Tables 1,2 and 3). This result corroborates the work of Ziółkowska and Wyszkowski [17], where contamination of soil with petroleum was reported not to adversely affect the vegetative development of edible plant by can lead to hydrocarbon accumulation in their foliar parts. The increase in concentration of petroleum hydrocarbons observed in the control may be due to trans-boundary contamination caused by horizontal flow of hydrocarbon or due human and animal activities on the research site Tables 1 and 2 . Moreover, Ohiri, et al. [18] reported an increase in heavy metal content of crude oil polluted and remediated agricultural soil and they attributed it to heavy metal presence in the petroleum. Their report also corroborates that of Chicarelli, et al. [19] where heavy metals like nickel and copper are present in petroleum through association with porphyrins.

Ingestion is the most common route of exposure of bioaccumulated toxic chemicals from edible plants to humans and other animals alike. However, most toxicants diffuse through the cell membrane in the non-ionized form, thus the degree of ionization is important in determining chemical absorptivity [20]. The increased toxicological parameters recorded in this study can be attributed to the ingestion and biotransformation of plant accumulated hydrocarbons and heavy metals by the liver and subsequent excretion via the renal processes of the kidney. (Table 4 and 5). The kidney has been responsible for excretion of toxic compounds but in the process of excretion, compounds that are soluble in lipids (e.g. aliphatic and polycyclic aromatic hydrocarbons) are easily reabsorbed through the renal tubule [21], thereby causing accumulation of toxic compounds in the kidney. This accumulation ultimately leads to impairment in renal function, resulting in increased urea and creatinine and destruction of kidney cells as noticed in rats fed T. occidentalis from bioaugmented and natural attenuated lots Table 4 and Figures $2 \mathrm{~b}$ and $2 \mathrm{c}$.

Toxic substances in the liver are biotransformed or secreted into the bile. However, biliary excretion of toxicants does not tantamount to elimination of such toxicants from the body. Reabsorption of biliary metabolites results into an enterohepatic cycle, thereby keeping the toxicants in the body and subjecting the livers to more stress conditions. This can lead to destruction of hepatic cells and subsequent elevation hepatic marker enzymes like alkaline phosphatase, aspartate and alanine aminotransferases in the serum. Transformation of toxicants by the liver may produce a potential carcinogen [22], which may subsequently lead to cell proliferation as noticed in the livers of rats fed $T$ occidentalis from the bioaugmented and natural attenuated lots Figure $3 \mathrm{~b}$ and $3 \mathrm{c}$. However, the slight increase in serum amylase activity observed in this study indicates minimal destruction of pancreatic cells. Table 5 and Figures $4 \mathrm{a}-4 \mathrm{c}$.

The pronounced reduction in packed cell volume and hemoglobin concentration recorded this study Table 6 may be as a result of destructions of the cells of the kidney and liver. This may cause an impairment in the secretion of erythropoietin thereby resulting to anaemia. Erythropoietin in the presence of colony-stimulating factors (CSF) regulates the differentiation of stem cell in the bone marrow, which ensures that erythrocyte precursor cells are converted to erythrocytes [21]. Aside neurological and developmental anomalies, exposure to elevated lead concentrations may lead to biochemical defects in both humans and animals. The resultant effect of such impairment may be on kidneys, gastrointestinal tract, joints and reproductive tissues, thereby affecting haemoglobin synthesis [23]. This tantamount to the observed decrease in packed cell volume and haemoglobin concentration recorded in rats fed $T$ occidentalis from natural attenuated and bioaugmented lots. Conclusively, the 
hydrocarbon and heavy metal concentration in crops harvested from crude oil polluted and remediated soil has to be evaluated before crops from such environment can be allowed for both human and animal consumption.

\section{References}

[1] D. W. Stevenson, Plant uptake of water and mineral nutrients from soil. Chicago: Encyclopedia Britannica, 2009

[2] Z. Yang and L. Zhu, "Performance of the partition-limited model on predicting ryegrass uptake of polycyclic aromatic hydrocarbons," Chemosphere, vol. 67, pp. 402-409, 2007. Available at: https://doi.org/10.1016/j.chemosphere.2006.09.008.

[3] A. Kipopoulou, E. Manoli, and C. Samara, "Bioconcentration of polycyclic aromatic hydrocarbons in vegetables grown in an industrial area," Environmental Pollution, vol. 106, pp. 369-380, 1999. Available at: https://doi.org/10.1016/s0269-7491(99)001074.

Leaf, "Wikipedia, the free encyclopedia, Retrieved from; http://en.wikipedia.org/wiki/leaves.," 2011.

FAO, "Food and agriculture organization minor oil crops," ed Rome: FAO Agricultural Services Bulletin, 1992, p. 94. AOAC, Official methods of analysis. Virginia, USA: Association of Official Analytical Chemists, 2006.

J. B. Jones Jr, "Collaborative study of the elemental analysis of plant tissue by direct reading emission spectroscopy," Journal of the Association of Official Analytical Chemists, vol. 58, pp. 764-769, 1975. Available at: https://doi.org/10.1093/jaoac/58.4.764.

[8] W. H. Marsh, B. Fingerhut, and E. Kirsch, "A sensitive method for the colourimetric determination of urea," Journal of Clinical Pathology, vol. 14, pp. $418-424,1961$.

[9] C. Slot, "Alkaline picric acid for determination of creatinine" Scand," The Journal of Clinical Investigation, vol. 17, pp. 381 - 387, 1965 .

[10] W. Hohenwallner, E. Wimmer, and R. Sommer, "Kinetic method for alpha-amylase determination in serum and urine," Journal of Clinc Chemistry and Biochemistry, vol. 17, pp. 349 - 352, 1979.

[11] P. Kind and E. King, "Estimation of plasma phosphatase by determination of hydrolysed phenol with amino-antipyrine," Journal of Clinical Pathology, vol. 7, pp. 322-326, 1954. Available at: https://doi.org/10.1136/jcp.7.4.322.

[12] S. Reitman and S. Frankel, "A colorimetric method for the determination of serum glutamic oxalacetic and glutamic pyruvic transaminases," American Journal of Clinical Pathology, vol. 28, pp. 56-63, 1957. Available at: https://doi.org/10.1093/ajcp/28.1.56.

[13] L. Corash, Laboratory haematology: Methods for the analysis of blood., In R. I. Handin, S. E. Lux and T. P. Stossel, (eds), Blood: Principles and practice of haematology. Lippincott Company, J. B: Philadelphia, 1995.

[14] H. J. Conn, Biological stains. In R. D. Lillie, 8th ed. Baltimore: Williams and Wilkins, 1969.

[15] G. L. Kumar and G. W. Gill, "Introduction to special stains. In: G. L. Kumar, and J. A. Kiernan, (eds.) Special stains and H\&E," ed Denmark: Dako Publication, 2010, pp. 16-42.

[16] D. B. Duncan, "Multiple range and multiple F tests," Biometrics, vol. 11, pp. 1-42, 1955. Available at: https://doi.org/10.2307/3001478.

[17] A. Ziółkowska and M. Wyszkowski, "Toxicity of petroleum substances to microorganisms and plants," Ecological Chemistry and Engineering, vol. 17, pp. 73-82, 2010.

[18] R. C. Ohiri, A. A. Uwakwe, and E. N. Onyeike, "Variations in mineral element and heavy metal contents of some vegetables grown on crude oil post-remediated agricultural soil," Journal European Chemical Bulletin, vol. 2, pp. 414 - 416, 2013.

[19] M. Chicarelli, C. Eckardt, C. Owen, J. Maxwell, G. Eglinton, R. Hutton, and A. Eaton, "Application of inductively coupled plasmamass spectrometry in the detection of organometallic compounds in chromatographic fractions from organic rich shales," Organic Geochemistry, vol. 15, pp. 267-274, 1990. Available at: https://doi.org/10.1016/0146-6380(90)90004-j.

[20] C. D. Klassen, M. O. Amdur, and J. Doull, The basic science of poison. Chicago: Encyclopedia Britainnica, 2009.

$\left.{ }_{21}\right] \quad$ J. Koolman and K. H. Roehm, "Colour atlas of biochemistry," 2nd ed New York: Thieme Stuttgart, 2005, pp. $330-331$

J. H. Ottaway, "The biochemistry of pollution," ed London: Edward Arnold Publication, 1980, pp. 12 - 20.

[23] S. K. Karri, R. B. Saper, and S. N. Kales, "Lead encephalopathy due to traditional medicines," Current Drug Safety, vol. 3, pp. 54-59, 2008. Available at: https://doi.org/10.2174/157488608783333907. 